\title{
Décret du 8 avril 1999 portant nomination de Mme Nicole Questiaux présidente de la Commission nationale de médeci- ne et de biologie de la reproduction et du diagnostic prénatal
}

\section{Arrêté du 8 avril 1999 portant nomination à la Commission nationale de médecine et de biologie de la reproduction et du diagnostic prénatal}

Par arrêté du secrétaire d'Etat à la santé et à l'action sociale en date du 8 avril 1999, sont nommés membres de la Commission nationale de médecine et de biologie de la reproduction et du diagnostic prénatal :

1. Pour chacune des sections de l'assistance médicale à la procréation et du diagnostic prénatal :

a) En qualité de représentant du Comité consultatif national d'éthique pour les sciences de la vie et de la santé, sur proposition de son président : M. le professeur Courtecuisse (Victor) ;

b) En qualité de représentante des associations familiales, sur proposition du président de l'Union nationale des associations familiales : Mme Lebatard (Chantal);

c) En qualité de médecin inspectrice d'une direction départementale des affaires sanitaires et sociales: Mme le docteur Dumont (Marie-Claude) ;

d) En qualité de pharmacien inspecteur d'une direction régionale des affaires sanitaires et sociales: M. Attal (Paul);

e) En qualité de haute personnalité scientifique : M. le professeur Sureau (Claude) ;

f) En qualité de spécialiste du droit de la filiation : Mme Paley-Vincent (Catherine) ;

g) En qualité de praticien ayant une formation ou une expérience particulière en génétique humaine, sur proposition de la Société française de génétique humaine : M. le professeur Lauras (Benoît) .

2. Pour la section de l'assistance médicale à la procréation :

A - Praticiens désignés sur proposition des organisations représentatives

a) En qualité de gynécologue-obstétricien et biologiste, sur proposition du groupe d'étude de la fécondation in vitro en France : 
M. le professeur Mares (Pierre);

M. Plouchart (Jean-Michel);

b) En qualité de biologiste de la reproduction, sur proposition de la Fédération des biologistes des laboratoires d'études de la fécondation et de la conservation de l'œuf : M. le professeur Sele (Bernard).

c) En qualité de clinicien et biologiste, sur proposition de la Fédération des centres d'étude et de conservation des œufs et du sperme humains :

M. le professeur Pontonnier (Francis); M . le Professeur Foliguet (Bernard).

$$
\text { B - Personnalités compétentes }
$$

d) En qualité de médecin choisi en raison de sa compétence dans le domaine de l'assistance médicale à la procréation avec tiers donneur : Mme le docteur Arnal (Françoise) ;

e) En qualité d'épidémiologiste ayant une expérience en médecine de la reproduction : M. le docteur Demouzon (Jacques) ;

f) En qualité de gynécologue-obstétricien et biologiste d'un établissement public de santé ayant une expérience dans le domaine de l'assistance médicale à la procréation :

M. le professeur Pouly (Jean-Luc);

M. le professeur Barrière (Paul);

g) En qualité de gynécologue-obstétricien d'un établissement de santé privé ayant une expérience dans le domaine de l'assistance médicale à la procréation : M. le docteur Hazout (André) ;

h) En qualité de directeur de laboratoire d'analyses de biologie médicale ayant une expérience dans le domaine de l'assistance médicale à la procréation : M. Menezo (Yves) ;

i) En qualité de médecin expérimenté en andrologie : M. le professeur Jardin (Alain) ;

j) En qualité de personnalité scientifique choisie en raison de sa compétence dans la recherche en matière d'assistance médicale à la procréation : Mme Mandelbaum (Jacqueline).

\section{Pour la section du diagnostic prénatal :}

A. - Praticiens désignés sur proposition des organisations représentatives

a) En qualité de praticiens ayant une expérience de diagnostic prénatal, sur proposition de l'Association française pour le dépistage et la prévention des handicaps de l'enfant :

M. le docteur Dingeon (Bernard) ;

Mme le docteur Francannet (Christine);

b) En qualité de médecin, sur proposition de l'Association des cytogénéticiens de langue française : Mme le docteur Leporrier (Nathalie) ;

c) En qualité de gyénécologue-obstétricien expérimenté en matière de prélèvements sur le fœtus, sur proposition du Collège national des gynécologues et obstétriciens français : M. le professeur Pucch (Francis) ;

d) En qualité de médecin sur proposition de la Société francophone de médecine foetale :

M. le professeur Dumez (Yves).

$\mathrm{B}$ - Personnalités désignées en raison de leur compétence

e) En qualité de pédiatre exerçant son activité en maternité : Mme le docteur Vial (Michèle);

f) En qualité de médecins expérimentés en échographie fœetale : 
M. le professeur Oury (Jean-François);

M. le docteur Broussin (Bernard);

g) En qualité de biologistes ayant une expérience particulière dans la réalisation d'examens de biologie fotale, dont l'un en biologie moléculaire :

M. le professeur Forestier (Fernand);

M. Vidaud (Michel) ;

h) En qualité de praticiens ayant une expérience particulière dans la réalisation d'examens de cytogénétique, dont l'un exerce dans le secteur public et l'autre dans le secteur privé :

M. le professeur Vekemans (Michel);

Mme le docteur Carles (Elisabeth);

i) En qualité de personnalité scientifique choisie en raison de sa compétence dans la recherche en matière de diagnostic prénatal : M. le professeur Goossens (Michel). 